\title{
Reconstruction of elbow by free fibular graft in a case of osteoclastoma of proximal ulna: a rare case
} report

\begin{abstract}
Osteoclastoma is a benign aggressive bone tumour. Most commonly it is seen in epiphysiometaphyseal region around knee and distal radius. Proximal ulna is a rare location for osteoclastoma. According to reports only 3 such cases have been reported in English literature. We report one such case of osteoclastoma of proximal ulna. Patient presented with painless, progressive swelling around elbow for 4 months. Resection of proximal ulna and elbow reconstruction was done using non vascularized free fibular graft. At two years follow up patient is tumour free and has functional range of movement in elbow. We are reporting the case because of its rare location and for the indigenous treatment modality of using free fibular graft for elbow reconstruction.
\end{abstract}

Volume 3 Issue I - 2015

\author{
Thejaswi SG \\ Sikkim Manipal University, India
}

Correspondence: Thejaswi SG, Sikkim Manipal University, India,Tel 09845138713, Email thejshah@yahoo.com

Received: June 26, 2015 | Published: August 21, 2015

Keywords: osteoclastoma, tumour, proximal ulna, tensor fascia lata

\section{Introduction}

Osteoclastoma also known as giant cell tumour is a benign aggressive tumour. It has a slight female preponderance and is seen in the age group of 20-40yrs. Osteoclastoma is an epiphysio-meta physial tumour commonly seen in distal end of femur, proximal end of tibia and distal end of radius. ${ }^{1}$ Treatment options include curettage with or without bone grafting, extended curettage, wide excision and reconstruction. We are reporting a case of osteoclastoma of proximal ulna. According to reports only few such cases have been reported in English literature. After evaluation tumour was resected En-bloc and joint was reconstructed using free fibular graft. This case is being reported for its rare location and the indigenous treatment modality carried out.

\section{Case history}

A 30 year old male presented with painless swelling around his right elbow for a period of 4 months (Figure 1). It was insidious in onset with gradual increase in size which later on started to affect his elbow movements. On examination a localised swelling measuring $5 \mathrm{x}$ $6 \mathrm{~cm}$ in posterior aspect of elbow was seen which was non tender, hard in consistency and was continuous with proximal ulna. The overlying skin was normal. Terminal part of extension and flexion of elbow joint was limited. X-ray showed a lytic lesion involving entire proximal ulna with soap bubble appearance with destruction of articular surface (Figure 2). FNAC was done to aid the diagnosis and it showed features suggestive of osteoclastoma.

Giving a tumour free upper limb along with a functional elbow joint was a challenging part. Hence treatment plan included en-bloc excision of proximal ulna with tumour and safe margin of normal appearing bone and to reconstruct the elbow joint using free fibular graft.

\section{Surgical procedure}

Under general anaesthesia tumour was exposed through posterior approach to elbow (Figure 3). Proximal ulna with tumour and $2 \mathrm{~cm}$ of normal bone was excised (Figure 4). Another team of surgeons harvested required length of free fibular graft from upper fibula from ipsilateral leg. Head of the fibula was shaped to match the olecranon process of ulna and tensor fascia lata was covered over fibular head to act as articular surface (Figure 5). Graft was fixed to ulna using semi tubular plate and a K-wire was put for temporary immobilisation of elbow in 90degree flexion. Triceps tendon was sutured to the graft at proximal end. Specimen was sent for histopathological examination which confirmed the diagnosis of Osteoclastoma (Figure 6). Immediate post op $\mathrm{X}$ ray confirmed the proper fixation.

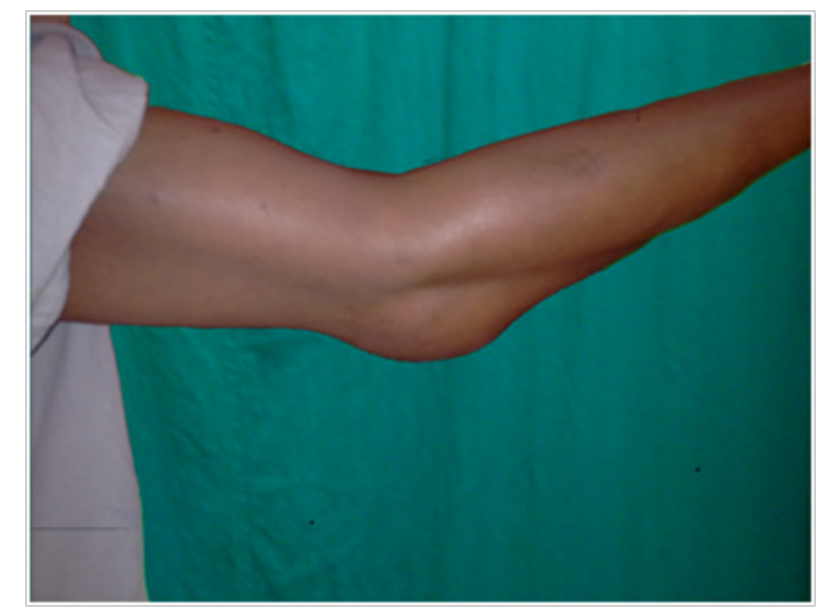

Figure I Swelling at the time of presentation.

\section{Follow up}

Post op period was uneventful. K wire was removed after 3 weeks and mobilisation of elbow was started. Patient was regularly followed up for 2 years for radiological union of the graft, functional outcome of the elbow and for possible recurrence or metastasis to lungs. Radiological union of the graft was seen as early as 4 months. At the end of 2 years patient is pain free and has a stable and functional elbow (Figure 7). No recurrence or metastasis of tumour seen. Donor site is also pain free and patient is walking comfortably. 


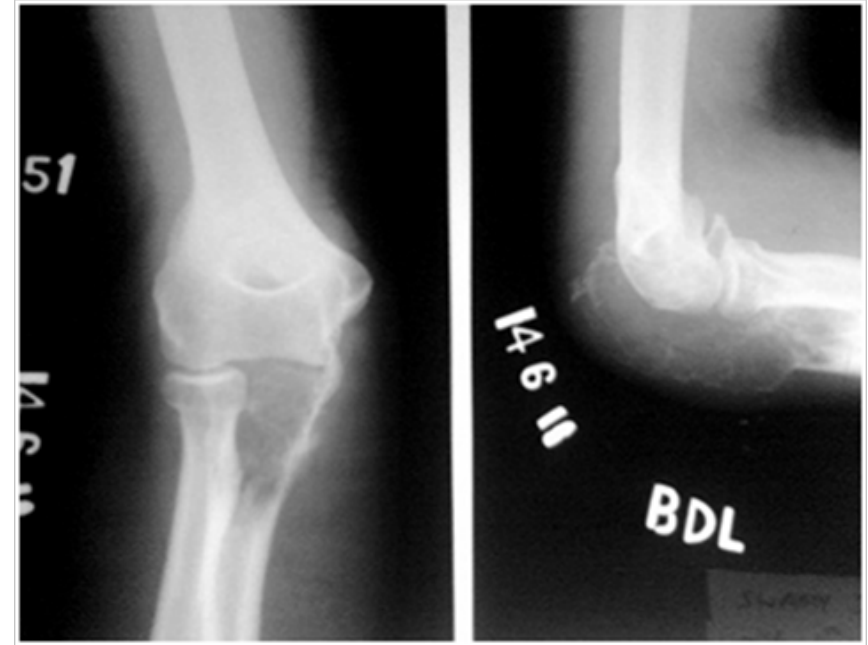

Figure $2 \mathrm{X}$-ray at the time of presentation.

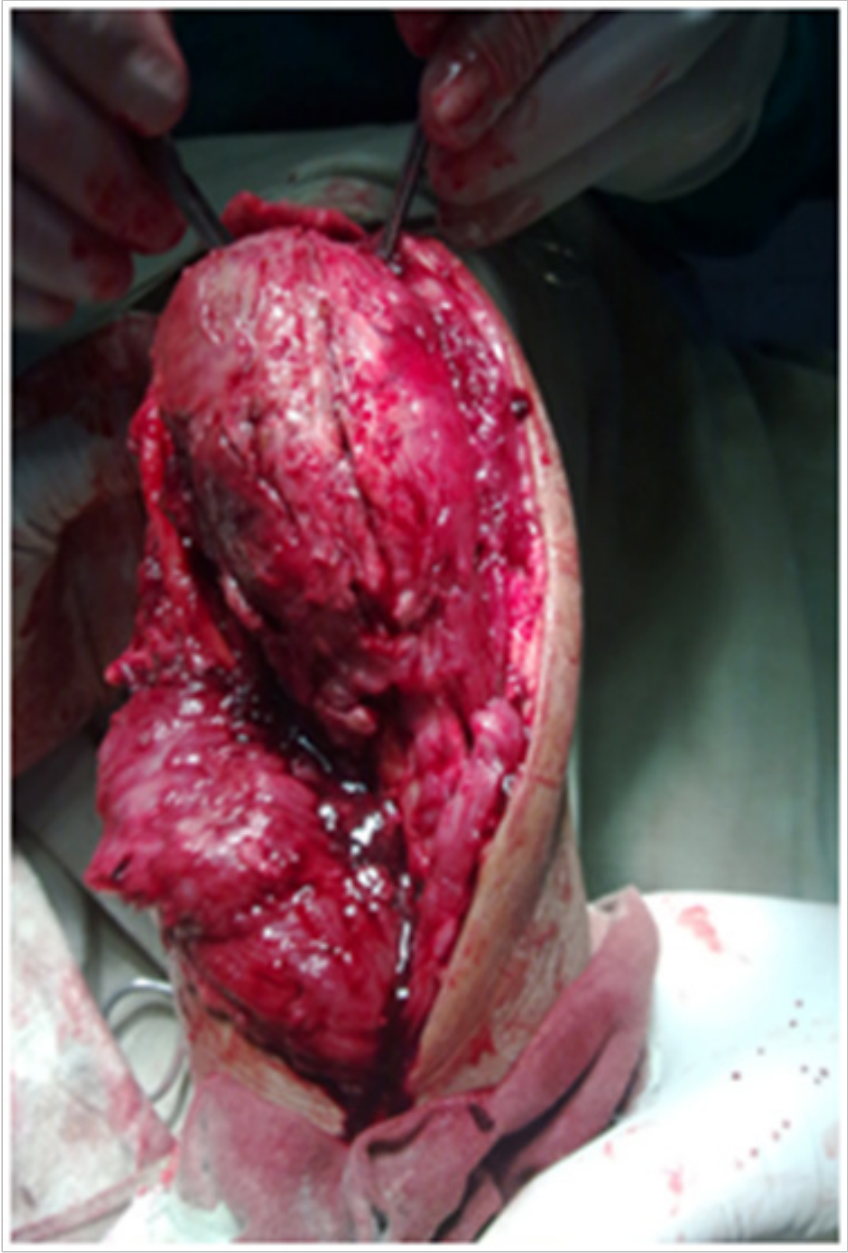

Figure 3 Per operative picture of tumour.

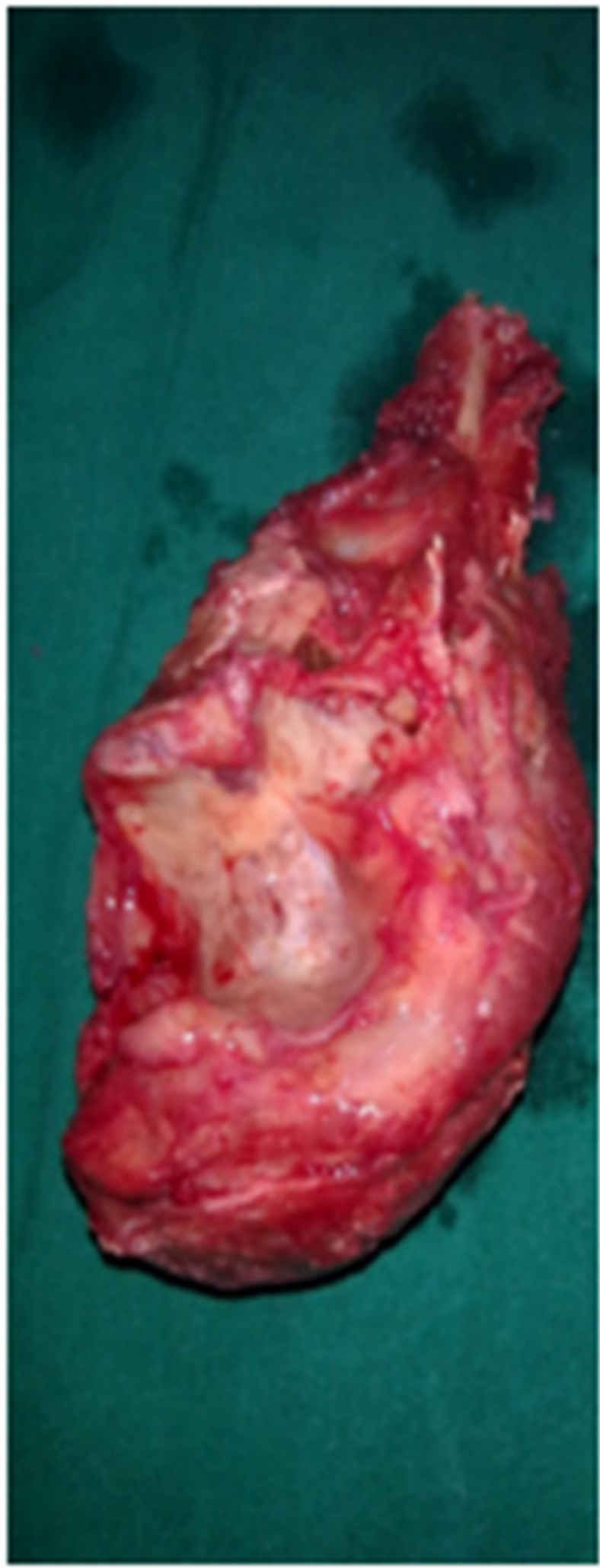

Figure 4 Excised tumour.

Citation: Thejaswi SG. Reconstruction of elbow by free fibular graft in a case of osteoclastoma of proximal ulna: a rare case report. MOJ Orthop Rheumatol. 20I5;3(I):279-282. DOI: 10.15406/mojor.2015.03.00087 


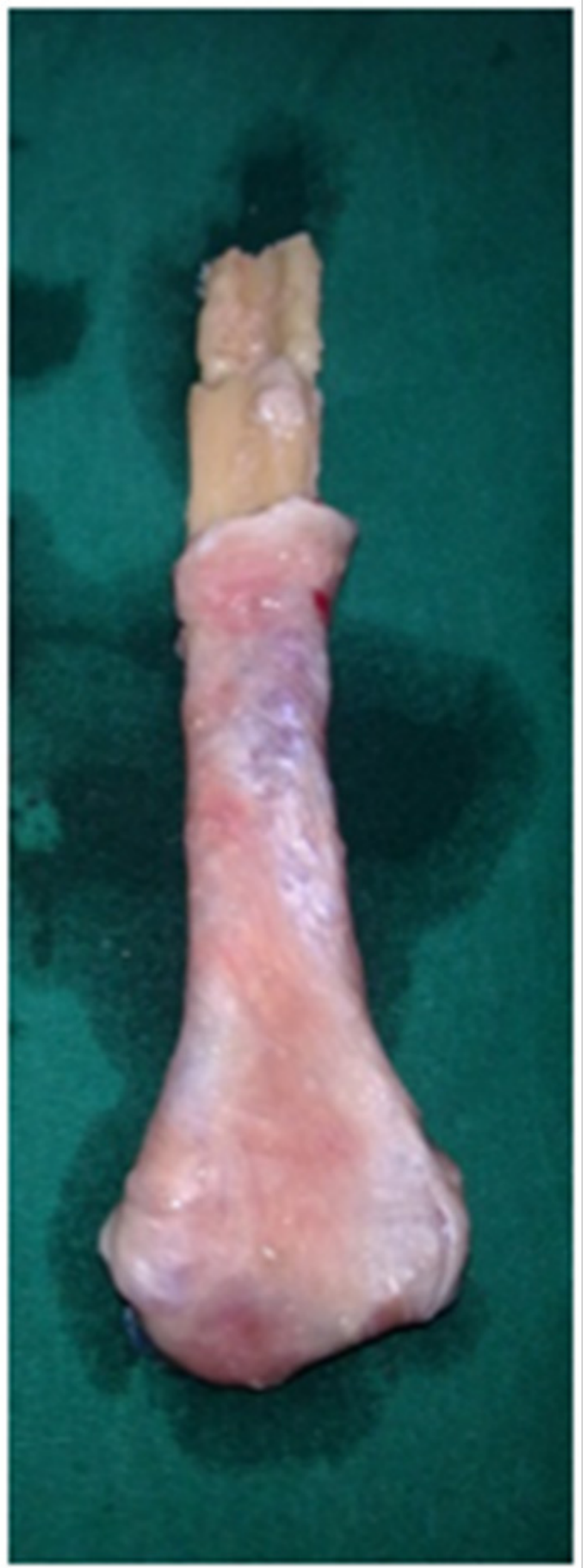

Figure 5 Prepared graft.

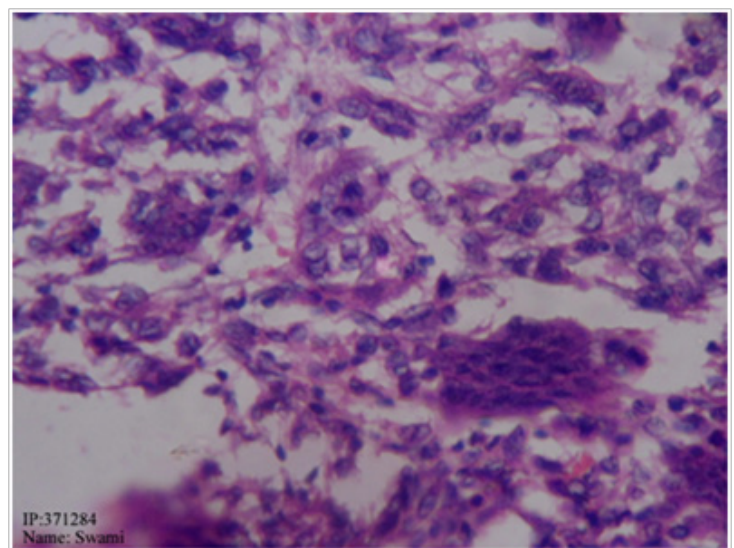

Figure 6 Histopathology showing gaint cell in cluster.

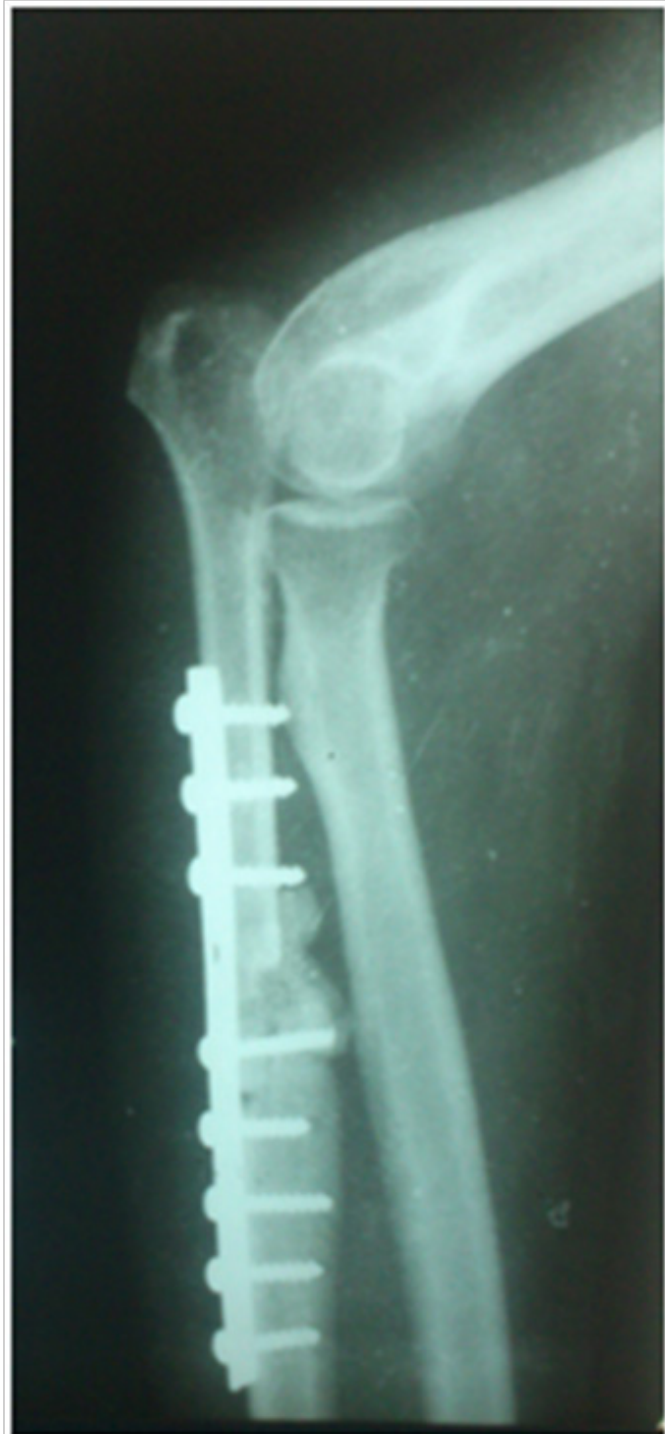

Figure 7 Follow up $\mathrm{X}$-ray after 2 years. 


\section{Discussion}

Osteoclastoma is a benign bone tumour but has aggressive course. It is seen after skeletal maturity with slight female preponderance. Epiphysio-metaphysical region around knee and distal end radius are favoured sites. ${ }^{1}$ Very few cases of osteoclastoma affecting proximal ulna have been reported. First case of osteoclastoma of proximal ulna in South East Asia was reported by Sanjy BK et al. ${ }^{2}$ And since then only one case has been reported in this region. To best of our knowledge this is only the third that is being reported from India and 5 th case ever being reported in English literature., ${ }^{2,3}$

Usual management of this tumour involves resection of the tumour or curettage and ablation of the cavity surface. This is feasible if the tumour is located in a long bone. In a small bone, excision and reconstruction is preferred. The challenge we faced was that the tumour involved proximal part of ulna along with elbow joint of the dominant upper limb of the patient. Hence it was necessary for us keep the bone and elbow joint free from tumour as well as give him a stable, functional elbow joint. In previous reported cases excision of proximal ulna along with arthrosdes is of elbow in functional position have been done. ${ }^{2,3}$ We planned to excise entire proximal ulna with tumour and $2 \mathrm{~cm}$ of visible normal bone and reconstruction of elbow joint using proximal free fibular graft along with tensor fascia lata coverage to act as articular surface.

Free fibular grafts have been used frequently in orthopedics as a graft to bridge long bony gaps and in very few cases to reconstruct joints. There have been reports of it being used to reconstruct elbow joint after resection of distal end of humerus. ${ }^{4-6}$ But reconstruction of ulnar component of elbow has rarely been attempted. Usui M et al. ${ }^{7}$ reported that the use of a free vascularized fibular graft including the head carried a risk of fibular head collapse. ${ }^{7}$ But such a thing has not been noticed yet by us till now in this case. In summery we were able to perform a limb saving procedure along with giving a stable functional joint using a free fibular graft. However further follow up and more research is needed in this regard to carry out this procedure as a regular treatment modality for tumours around elbow.

\section{Consent}

The patient has given informed consent for the case report to be published.

\section{Authors' contributions}

KK, MS, TSG were involved in diagnosing and planning of treatment as well as they were the operating team of surgeons for this case. TSG is involved in data collection and writing the manuscript.

\section{Acknowledgments}

None.

\section{Conflicts of interest}

The authors declare there is no conflict of interest.

\section{References}

1. Unni KK, Carrie YI. Dahlin's bone tumors: general aspects and data on 10,165 cases. 6 th ed. 2014:225-226.

2. Sanjay BK, Nagi ON, Gupta BD. Giant cell tumour of the proximal end of the ulna. Arch Orthop Trauma Surg. 1991;110(4):208-209.

3. Minhas MS, Mehboob G. Giant cell tumour of the proximal ulna. J Coll Physicians Surg Pak. 2010;20(6):416-418.

4. Barnea Y, Amir A, Shlomo D, et al. Free fibula flap elbow-joint hemiarthroplasty reconstruction for chronic osteomyelitis of the distal humerus. J Reconstr Microsurg. 2006;22(3):167-171.

5. Kimura K, Tatezaki S, Ishii T, et al. Hemiarthroplasty of the Elbow with a Vascularized Fibular Graft after Excision of Ewing's Sarcoma of the Proximal Ulna: a Case Report. Jpn J Clin Oncol. 2002;32(10):430-434.

6. Gianoutsos MP, Marsden FW, McCarthy SW, et al. Ulnar adamantinoma: en bloc excision and fibular osteoseptocutaneous free flap reconstruction. J Hand Surg Am. 1994;19(3):495-499.

7. Usui M, Murakami T, Naito $T$, et al. Some problems in wrist reconstruction after tumor resection with vascularized fibular-head graft. J Reconstr Microsurg. 1996;12(2):81-88. 